\title{
Current Use of Noninvasive Hemoglobin Monitoring in Anesthesia
}

\author{
Ji-Hyun Lee $\cdot$ Yong-Hee Park $\cdot$ Jin-Tae Kim
}

Published online: 17 July 2014

(c) Springer Science + Business Media New York 2014

\begin{abstract}
Modern technology enables clinicians to assess hemoglobin levels noninvasively and continuously. Some instruments also provide concentrations of other types of hemoglobin, such as methemoglobin and carboxyhemoglobin. Such information obtained immediately and noninvasively can be helpful in the management of critically ill patients or those undergoing surgery with bleeding. However, the accuracy of noninvasive hemoglobin measurements should be evaluated before clinical application, particularly for transfusion decisions. Clinicians should be aware of the performance of continuous noninvasive hemoglobin monitoring devices during anesthesia and of the various factors that influence their accuracy. This review considers the accuracy of current continuous noninvasive hemoglobin monitoring and its clinical use.
\end{abstract}

Keywords Noninvasive $\cdot$ Hemoglobin $\cdot$ Transfusion

Ji-Hyun Lee and Yong-Hee Park equally contributed to this article as the 1 st author.

\section{J.-H. Lee · J.-T. Kim ( $\varangle)$}

Department of Anesthesiology and Pain Medicine, Seoul

National University Hospital, 101 Daehak-ro, Jongno-gu,

Seoul 110-744, South Korea

e-mail: kimjintae73@dreamwiz.com

J.-H. Lee

e-mail: muslab@hanmail.net

\section{Y.-H. Park}

Department of Anesthesiology and Pain Medicine, Chung-Ang

University Hospital, Seoul, South Korea

e-mail: dada822@naver.com

\section{Introduction}

Continuous noninvasive hemoglobin monitoring devices allow clinicians to identify hemoglobin levels, one of the determinants of oxygen delivery to the tissue, without sampling or delay. The concept of "hemoglobin monitoring" is possible because of the continuous and noninvasive characteristics of the device. Hemoglobin levels can be monitored continuously similar to blood pressure and heart rate during anesthesia. This may help clinicians detect occult bleeding or prepare for a transfusion. A surgical procedure itself can cause acute and massive bleeding that may threaten the patient's oxygen delivery. When hemoglobin decreases to $<6-7 \mathrm{~g} / \mathrm{dL}$, a transfusion is usually recommended. The accuracy of the noninvasive hemoglobin value $(\mathrm{SpHb})$ should be guaranteed in patients who need a transfusion. Various clinical factors can affect measurement accuracy during diverse clinical conditions. Therefore, it is prerequisite to understand factors that affect accuracy for proper use of a noninvasive hemoglobin monitoring system. In this review, we consider the current use of noninvasive hemoglobin monitoring systems and various conditions influencing their accuracy.

\section{Currently Available Devices for Noninvasive Hemoglobin Monitoring}

Masimo Corporation

A noninvasive hemoglobin monitoring device was developed from a pulse oximeter. Pulse oximetry measures oxygen saturation noninvasively using red $(660 \mathrm{~nm})$ and infrared $(950 \mathrm{~nm})$ light-emitting diodes. However, inaccuracy usually occurs in the presence of dyshemoglobins 
(carboxyhemoglobin or methemoglobin) or at a low hemoglobin concentration [1, 2]. In 2005, Masimo Corporation released the $\mathrm{Rad}-57$ device, which uses multiple wavelengths of light and pulse CO-oximetry technology. The eight wavelengths in the 500-1,300 nm range enable the measurement of fractional hemoglobin. Transmitted light has different optical densities at different wavelengths, which are analyzed and converted to a digital signal using a proprietary algorithm [2]. This technology was extended in 2008 to measurement of total hemoglobin concentration. Currently available devices are the Radical-7, a continuous monitoring bedside device, and the Pronto-7, a handheld spot-check device [3•]. Most studies have focused on the performance of these noninvasive hemoglobin measurement devices manufactured by Masimo Corporation. New software and probes to improve the accuracy of measurements are currently under development.

\section{OrSense Products}

The Rainbow technology has been revealed as inaccurate under poor perfusion conditions [4]; OrSense developed another noninvasive hemoglobin monitoring devices to overcome this limitation, the NBM-200 and NBM-200MP (OrSense, NesZiona, Israel). NBM-200 is spot-check device and analyze pulse rate and hemoglobin value. It uses differential light absorption before and after blood flow obstruction in a finger using a ring-shaped finger sensor that is temporarily squeezed to over-systolic pressure $[4,5]$. This technology is known as occlusion spectroscopy and has been used to noninvasively measure glucose concentration. The release of blood flow after occlusion generates a strong optical signal that is tenfold greater than the pulse signal [6]. The lights in wavelength ranging from 600 to $950 \mathrm{~nm}$ are emitted from the ten spectrally stabilized emitters and detected within sensor, and the hemoglobin concentration can be measured by analyzing the signals at these wavelengths [7]. NBM200MP additionally displays plethysmography and oxygen saturation of hemoglobin, based on the association of lowperfusion oximetry combined with occlusion spectroscopy [4]. Several studies of the OrSense products have been conducted, but have shown inconsistent results with a wide variation in biases. In addition, limited data involve unstable patients with bleeding. Therefore, further studies are required if this device is to be used in clinical practice, particularly in unstable patients with bleeding.

\section{Haemospect}

Haemospect (MBR Optical Systems, Herdecke, Federal Republic of Germany) is a spectroscopic device that uses visible and near-infrared lights. It was developed from the Mediscan 2000 system and was first marketed in 2008. The Haemospect sensor has light-emitting and -detecting silica fibers that are $250 \mu \mathrm{m}$ apart. Halogenated white light is used and the reflected light is analyzed for spectra in the $350-1,020 \mathrm{~nm}$ range. Then, the system calculates the concentration and path length of light between the lightemitting and -receiving fibers using the non-linear Levenberg-Marquardt fitting algorithm, and the hemoglobin concentration is calculated. Although two studies showed a good correlation with actual total hemoglobin $(\mathrm{tHb})$ in pediatric patients $[8,9]$, limited data are available regarding its use during anesthesia.

\section{Evaluation of the Accuracy of Noninvasive Hemoglobin Monitoring}

During clinical practice, various conditions and patient factors can affect the accuracy of noninvasive hemoglobin measurement. These factors are perfusion state, the hemoglobin level itself, a large volume shift, type of infused fluid, and age of the patient. Many studies have used the correlation coefficient, bias, precision, and limits of agreement (LOA) to evaluate the accuracy of devices. However, none of these methods can perfectly evaluate accuracy from the clinical perspective.

The correlation coefficient indicates simply the strength and direction of the linear relationship between two variables but provides no information on the clinical implication of a specific value. Bias is the degree of closeness to the actual value and is defined as the average of paired differences for all measurements. Precision is the degree to which repeated measurements yield identical results under unchanged conditions and is manifested as the standard deviation of the difference in paired measurements. The LOA represent the dispersion of data around the bias line, which is calculated from the bias \pm 1.96 standard deviations.

Although bias is used widely to compare two devices, low bias does not necessarily infer an accurate instrument [10••]. Even if data are distributed widely from the actual value but the spread above and below 0 is approximately identical, the bias is 0 . Absolute bias is occasionally used to reduce this problem. In addition, the Bland-Altman analysis is designed to measure fixed, not proportional, bias. Therefore, if there is a relationship between the $\mathrm{tHb}$ and $\mathrm{SpHb}$, LOA are not reliable [10••].

A simple method to analyze trending ability is based on regression analysis and concordance, and was first described by Perrino et al. [11]. It is used when evaluating the accuracy of cardiac output $(\mathrm{CO})$ monitoring. The test $\Delta \mathrm{CO}$ 
was plotted against the reference $\Delta \mathrm{CO}$ on a four-quadrant scatter plot [12]. The concordance rate was simply a measure of the number of data points that fell into one of the two quadrants of agreement. Another method to evaluate trending is a polar plot, which is described in Critchley's review [12].

Because the clinical meaning of SpHb accuracy differs according to $\mathrm{tHb}$ level, the clinical error grid has been applied to evaluate the accuracy of $\mathrm{SpHb}[13 \bullet, 14,15 \bullet]$. This method was first used to evaluate blood glucose monitoring devices. Hemoglobin and blood glucose levels have similar aspects in terms of the clinical meaning of values that differ from the real values (when the values are out of the normal range, the meaning of its value is greater). A clinical error grid is a practical method of evaluating the clinical significance of $\mathrm{SpHb}$.

\section{Accuracy in Various Clinical Situations}

During clinical practice, $\mathrm{SpHb}$ can be applied to patients of different ages (adults, children, infants, or neonates) in various locations [operating room, intensive care unit (ICU), emergency room, or outpatient ward], during different surgical procedures, in healthy or ill individuals, and under different physiological conditions (poor peripheral perfusion, active bleeding, or rapid fluid administration). Understanding the performance of noninvasive hemoglobin monitoring devices under diverse conditions facilitates their use during anesthesia.

\section{Pediatric Patients}

$\mathrm{SpHb}$ data in pediatric patients are limited. Various pediatric sensors for Masimo devices are available, which are based on patient weight (Rainbow R20 is for $10-50 \mathrm{~kg}$, Rainbow R125L is for $<3 \mathrm{~kg}$, and the Rainbow $\mathrm{R} 120 \mathrm{~L}$ is for $3-30 \mathrm{~kg}$ ). The degree of $\mathrm{SpHb}$ accuracy in pediatric patients who underwent neurosurgery or preoperative phlebotomy is similar to that of adults $[16,17]$. In neonates, the benefit of $\mathrm{SpHb}$ may be greater than in adults or large children because neonates have a limited blood volume. Even a small blood sample can cause anemia if $\mathrm{Hb}$ measurements are repeated. One report in a neonatal ICU showed a clinically acceptable accuracy and correlation with tHb [18]. However, the accuracy and usefulness of the Radical-7 are questionable in multivisceral pediatric transplant patients [19]. In summary, although $\mathrm{SpHb}$ offers a clinically acceptable correlation with $\mathrm{tHb}$ and the benefit of a continuous trend monitor, critical decisions should not be made in pediatric patients based on this device alone.
Location

\section{Operating Room}

The correlation coefficient during vascular surgery is lower than during other types of surgery [20]. The type of surgery can influence the accuracy of a noninvasive hemoglobin monitoring device; moreover, intraoperative physiology may also affect its performance.

Potentially Hemorrhagic Abdominal or Pelvic Surgery Applegate et al. [21•] evaluated the performance of the Radical-7 in gynecologic, urologic, and general abdominal surgeries with mean estimated blood loss of $760 \mathrm{~mL}$. That study of 91 surgical patients showed a weak correlation between $\mathrm{SpHb}$ and $\mathrm{tHb}$. In addition, $\mathrm{SpHb}$ was out of $1 \mathrm{~g} / \mathrm{dL}$ of $\mathrm{tHb}$ in $48.6 \%$ of the measurement pairs. Those authors found that several characteristics influenced bias. The bias was larger in patients with blood loss $>1,000 \mathrm{~mL}$, hemoglobin $<9.0 \mathrm{~g} / \mathrm{dL}$, or any intraoperative transfusion, whereas the bias was reduced during deep anesthesia. Increased bias in patients who require a transfusion may be an obstacle to use of $\mathrm{SpHb}$ monitoring for the decision to perform a transfusion.

The accuracy of the Radical-7 was compared with that of HemoCue $(\mathrm{cHb})$ in a study of patients scheduled for potentially hemorrhagic major urologic surgery [22]. The correlation coefficient was lower and LOA were wider for the Radical-7 than those for the $\mathrm{cHb}$. In addition, the percentage of outliers (defined as the absolute bias $>1 \mathrm{~g} / \mathrm{dL}$ ) for the Radical-7 was significantly higher than that for the cHb (46 vs. $16 \%, P<0.05$ ). Therefore, the authors concluded that $\mathrm{SpHb}$ monitoring with the Radical-7 gives a lower reading than that of the $\mathrm{cHb}$ in terms of assessing hemoglobin concentration during hemorrhagic surgery. The accuracy of noninvasive continuous hemoglobin monitoring is lower in patients with bleeding than in healthy individuals.

Cesarean Section (CS) Butwick et al. [23] evaluated the accuracy of the Radical-7 in 50 healthy term patients undergoing elective CS under neuroaxial anesthesia. Measurements were obtained at the following time points: baseline (before preload during the preoperative period), during the immediate postoperative period (within $10 \mathrm{~min}$ of arrival in recovery), and $24 \mathrm{~h}$ after completion of surgery. The bias series and LOA are demonstrated in Table 1 . SpHb tended to be higher than $\mathrm{tHb}$ with a greatest difference 24-h post-CS. Considering that no patient experienced significant obstetric hemorrhage, the variable bias and wide LOAs may limit the clinical utility of the Radical-7 for assessing tHb and making transfusion decisions in patients undergoing elective CS. 


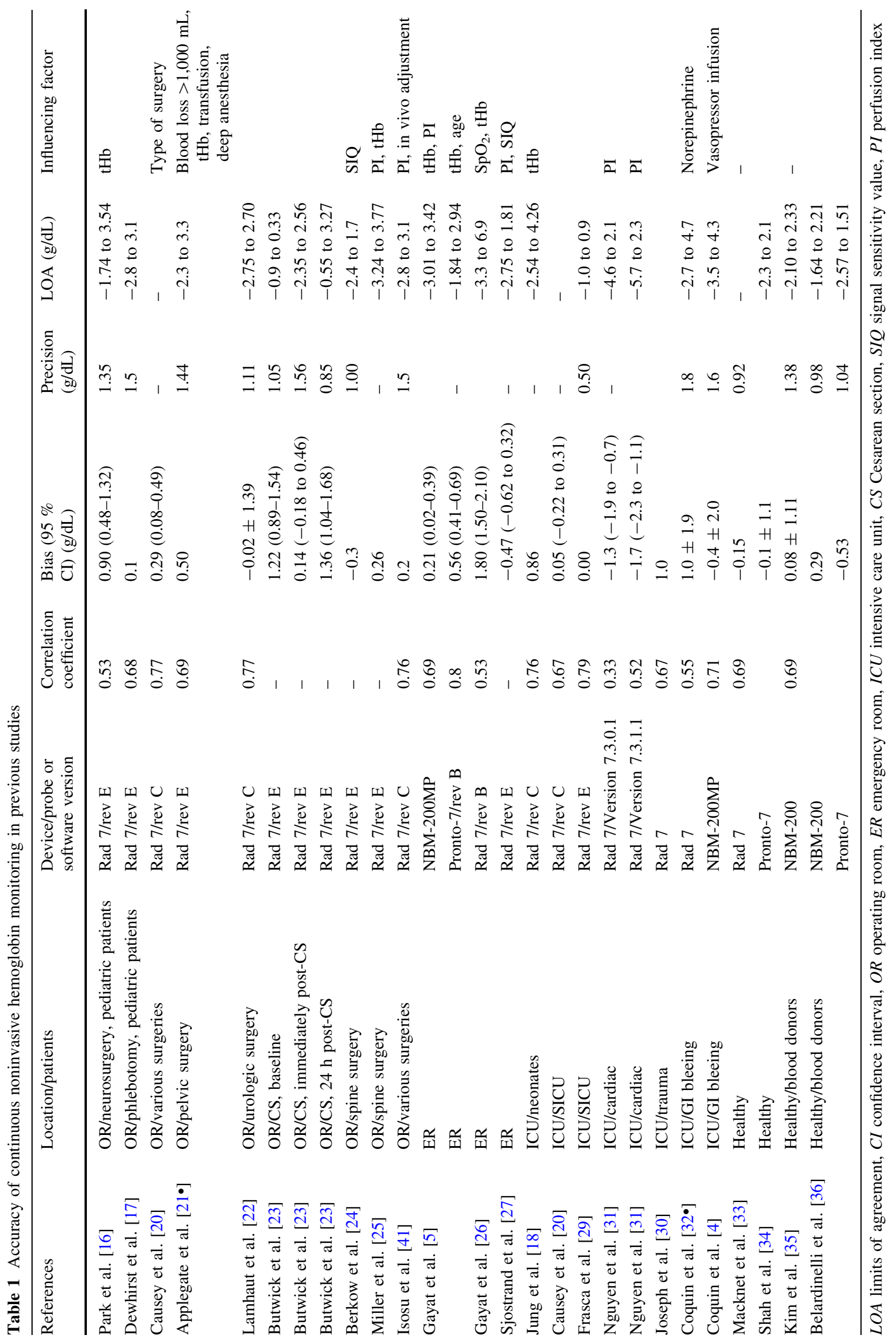


Spine Surgery The first study of spinal surgery reported that the Radical-7 has clinically acceptable accuracy [24]. However, a subsequent spinal surgery study showed a relatively large LOA and the effects of various other factors [25]. In Miller's study, the Radical-7, tHb, and $\mathrm{cHb}$ were compared. A total of 78 paired $\mathrm{Hb}$ samples were collected from 20 patients and analyzed. Overall, $61 \%$ of the $\mathrm{SpHb}$ values corresponded with the $\mathrm{tHb}$ values by $<1.5 \mathrm{~g} / \mathrm{dL}$. The authors suggested that $\mathrm{SpHb}$ monitoring is expected to facilitate transfusion management by providing additional and continuous data. However, the large LOA and other influencing factors should be considered.

Neurosurgery The accuracy of the Radical-7 during neurosurgery has been evaluated in pediatric patients $<12$ years old [16]. Overall, the difference in $\mathrm{SpHb}$ and $\mathrm{tHb}$ was $<1.5 \mathrm{~g} / \mathrm{dL}$ in $87(73.1 \%)$ measurement pairs. If the patient was in a hypovolemic state, the authors infused either colloid or red blood cells based on their protocol. Subsequently, the changes in $\mathrm{SpHb}$ and $\mathrm{tHb}(\Delta \mathrm{SpHb}$ and $\Delta \mathrm{tHb})$ before and after volume resuscitation were compared. The concordance rate (a measure of the number of data points that are in one of the two quadrants of agreement) using a four-quadrant plot was $94.4 \%$, and the correlation coefficient between paired measurements of $\Delta \mathrm{SpHb}$ and $\Delta \mathrm{tHb}$ after volume resuscitation was $0.87(P<0.001,95 \%$ confidence interval, 0.84-0.92). However, the bias was influenced by the tHb concentration. Although $\mathrm{SpHb}$ can be useful as a trend monitor during surgery even immediately after administration of intravascular volume expanders, the clinician should be cautious when $\mathrm{SpHb}$ alone is used to make transfusion decisions.

\section{Emergency Room}

Noninvasive hemoglobin monitoring is also valuable in emergency care. The first study from the emergency room using the Radical-7 concluded that $\mathrm{SpHb}$ is systemically biased and insufficiently reliable to guide transfusion decisions because of the large bias and LOA [26]. SpHb could not been obtained with the Radical-7 in 24 of 300 patients who were significantly older and had lower $\mathrm{SpO}_{2}$ and diastolic blood pressure. Another study using the Radical-7 in 30 patients with stable hemodynamics in an emergency room demonstrated improved bias and LOAs. Although its accuracy seemed to be improved compared to a previous report, it had difficulty predicting $\mathrm{tHb}$ with a single $\mathrm{SpHb}$ value [27]. Gayat et al. [5] evaluated the performance of two noninvasive hemoglobin monitoring devices, the Pronto-7 and the NBM-200MP, in 300 patients in a stable condition. Although the NBM-200MP was associated with higher variability and a larger LOA compared to those of the Pronto-7, both devices had large LOA, making their clinical usefulness debatable. Furthermore, no significant fluid shift condition, blood loss, or active bleeding was observed. In recent study, Moore et al. [28] reported that the Radical-7 device failed to detect $\mathrm{Hb}$ values in $34 \%$ of cases and the $\mathrm{SpHb}$ showed poor correlation with $\mathrm{tHb}$ in severely injured trauma patients in emergency room. More studies that include hemodynamically unstable patients are required for clinical use of noninvasive hemoglobin monitoring systems in the field of emergency care. In addition, the problems associated with movement of the probe in conscious patients should be considered.

\section{$I C U$}

ICU patients have much in common with patients under anesthesia. One study of patients in the general ICU showed that $\mathrm{SpHb}$ measured with the Radical-7 has absolute and trending accuracy similar to the widely used invasive $\mathrm{cHb}$ [29]. However, in that study, $<10 \%$ of the measurements were performed in patients with a hemoglobin $<8 \mathrm{~g} / \mathrm{dL}$, and no patient was actively bleeding. Other studies on patients with bleeding failed to show high accuracy of the Radical-7. Data were not recorded in severely injured ICU patients $13.5 \%$ of the time. About $58 \%$ of their data pairs had a difference $>1 \mathrm{~g} / \mathrm{dL}$, and $>32 \%$ had a difference $>2 \mathrm{~g} / \mathrm{dL}$ [30]. Another report on 41 cardiac ICU patients showed a poor correlation and wide LOA between $\mathrm{SpHb}$ (Radical-7) and $\mathrm{tHb}$ [31]. Additionally, $40 \%$ of the patients received norepinephrine. That study compared two versions of the Radical-7 software (V 7.3.0.1 vs. V 7.3.1.1), and found no difference. The authors suggested that noninvasive $\mathrm{Hb}$ monitoring technology should be improved before its use in anesthesiology or the intensive care setting. Determining hemoglobin using a noninvasive hemoglobin monitoring device in ICU patients with severe gastrointestinal bleeding appears inaccurate for clinical decision making [4, 32•]. In ICU patients admitted for gastrointestinal hemorrhage, the NBM-200MP lacked accuracy compared with cHb regardless of the measurement site [4]. When a study of the Radical-7 in a similar situation was performed, its clinical performance and accuracy were also not acceptable. The proportion of inaccurate measurements was $56 \%$ for noninvasive hemoglobin monitoring devices compared with $15 \%$ for $\mathrm{cHb}$ [32•]. In summary, accuracy seems to decrease in ICU patients with bleeding. Therefore, clinicians should be cautious when interpreting the $\mathrm{SpHb}$ value for management of ICU patients.

\section{Other Situations}

The $\mathrm{SpHb}$ value appears to be more accurate in healthy individuals [33, 34]. SpHb measured using the Radical-7 was accurate within $1.0 \mathrm{~g} / \mathrm{dL}$ compared with that of $\mathrm{tHb}$ 
measurements in healthy subjects undergoing hemodilution [33]. The Pronto-7 provided accuracy similar to the $\mathrm{cHb}$, invasive point-of-care device, in healthy patients [34]. In contrast, the accuracy was not optimal for blood donor screening.

The use of noninvasive $\mathrm{Hb}$ measurement for blood donor screening is controversial. Kim et al. [35] evaluated the usefulness of the NBM-200 for blood donor screening and concluded that wrong decisions could occur when deciding eligibility for blood donation using this device. Another reports of blood donor screening using the NBM200 are available [36, 37]. Pinto et al. compared performance of fingerstick sampling and NBM-200, and significantly lower level of percentage error was found in NBM200 compared to reference value $(\mathrm{tHb})$. On the other hand, Belardinelli et al. evaluated the accuracy of the NBM-200 and Pronto-7 and assessed whether they could enhance recruitment and retention of blood donors. However, both devices, particularly the Pronto-7, had lower specificity and sensitivity and the risk of inappropriate donation.

During pregnancy, noninvasive hemoglobin monitoring can provide additional benefit to detect gestational anemia or obstetrical hemorrhage. Hadar et al. [38] evaluated accuracy of NBM-200 in pregnant women. The mean bias and standard deviation of NBM-200 were 0.1 and 0.86 $\mathrm{g} / \mathrm{dL}$, and the $\mathrm{SpHb}$ strongly correlated to $\mathrm{tHb}$ from venous sampling. Thus, authors concluded NBM-200 may serve an important role in obstetrics allowing easy, rapid, and accessible evaluation of hemoglobin.

In another circumstance, such as during hemodialysis, $\mathrm{SpHb}$ was used to estimate the changes of blood volume [39]. Although absolute values of $\mathrm{SpHb}$ using Radical-7 were poorly correlated with $\mathrm{Hb}$ measured by Crit-Line, inline device that monitors blood volume changes during hemodialysis, the changes of blood volume determined from equation using $\mathrm{SpHb}$ showed good correlation with the changes measured by Crit-Line. This study emphasized the trending ability of the $\mathrm{SpHb}$.

\section{Clinical Factors Affecting SpHb Accuracy}

The accuracy of SpHb can be influenced by many factors, such as poor peripheral perfusion state, vasoconstrictor infusion, low hemoglobin level, and rapid fluid administration.

\section{Peripheral Perfusion}

Perfusion Index (PI) Value Obtaining the $\mathrm{SpHb}$ value with a PI $<1.4$ is not recommended by the Radical-7 manufacturer. The PI value has a definite influence on $\mathrm{SpHb}$ accuracy. One study showed that digital nerve block resulting in an increase of digital perfusion can increase PI, and this can increase accuracy of $\mathrm{SpHb}$ [40]. There is a tendency for $\mathrm{SpHb}$ to overestimate $\mathrm{tHb}$ when the PI is $>1.4$ $[25,41]$. Another report showed a similar pattern when PI was $>7.0$ [42]. Accuracy increases when the PI value is $>2.0$, and $\mathrm{SpHb}$ underestimates $\mathrm{tHb}$ when PI is $<2.0$ [27]. This pattern was also observed in cardiac ICU patients [31]. SpHb-tHb difference appears to increase with a higher PI (Fig. 1a). Accuracy increases and variability decreases with higher PI values (>4.0) [25]. The NBM200MP also showed a similar relationship between PI and the $\mathrm{SpHb}-\mathrm{tHb}$ difference value, but the Pronto-7 value was not influenced by PI [5].

Use of Norepinephrine In addition to accuracy of $\mathrm{SpHb}$, another important issue is data availability. If perfusion to peripheral tissue is poor, data availability can be limited. The number of unavailable measurements related to inadequate perfusion is significantly higher in patients receiving norepinephrine (42\% Radical-7 vs. $15 \% \mathrm{cHb}$ ) [32•]. Accordingly, vasoconstrictor administration can affect the usefulness of noninvasive hemoglobin monitoring.

Peripheral Vascular Disorders Any condition that obstructs peripheral perfusion can affect the accuracy and availability of data. Vasospastic diseases such as Raynaud's disease also lead to inappropriate $\mathrm{SpHb}$ values.

\section{Total Hemoglobin}

The differences between $\mathrm{SpHb}$ measured by the Radical-7 and the $\mathrm{tHb}$ value appear to be inversely correlated with higher $\mathrm{tHb}[16,21 \bullet, 25]$ (Fig. 1b). For example, the bias for all measurements was $0.26 \mathrm{~g} / \mathrm{dL}$ (95 \% LOA: -3.24, 3.77), whereas the estimated bias for $\mathrm{SpHb}$ values $>12 \mathrm{~g} / \mathrm{dL}$ was -1.64 (95 \% LOA: -4.58, 1.29) in patients undergoing spinal surgery [25]. Therefore, $\mathrm{SpHb}$ tends to overestimate $\mathrm{tHb}$ when $\mathrm{tHb}$ is low. This wrong information can lead to the clinician falsely believing that a patient's hemoglobin level is normal even when a transfusion is actually required. The Pronto-7 and NBM-200MP also showed similar results in that biases were inversely correlated with $\mathrm{tHb}$ [5]. Another study of the Radical-7 in neonates reported that the accuracy decreases dramatically when the Hb level is $>18 \mathrm{~g} / \mathrm{dL}$ [18].

\section{Fluid}

One report found that the accuracy of noninvasive hemoglobin monitoring depends on the type of infused fluid [42]. In healthy volunteers, administration of lactated Ringers' solution increases the $\mathrm{SpHb}-\mathrm{tHb}$ value by $7 \%$, whereas starch decreases it by $4.3 \%$. Another study showed that $\mathrm{SpHb}$ tends to change more than $\mathrm{tHb}$ during fluid infusion with crystalloid [43]. 

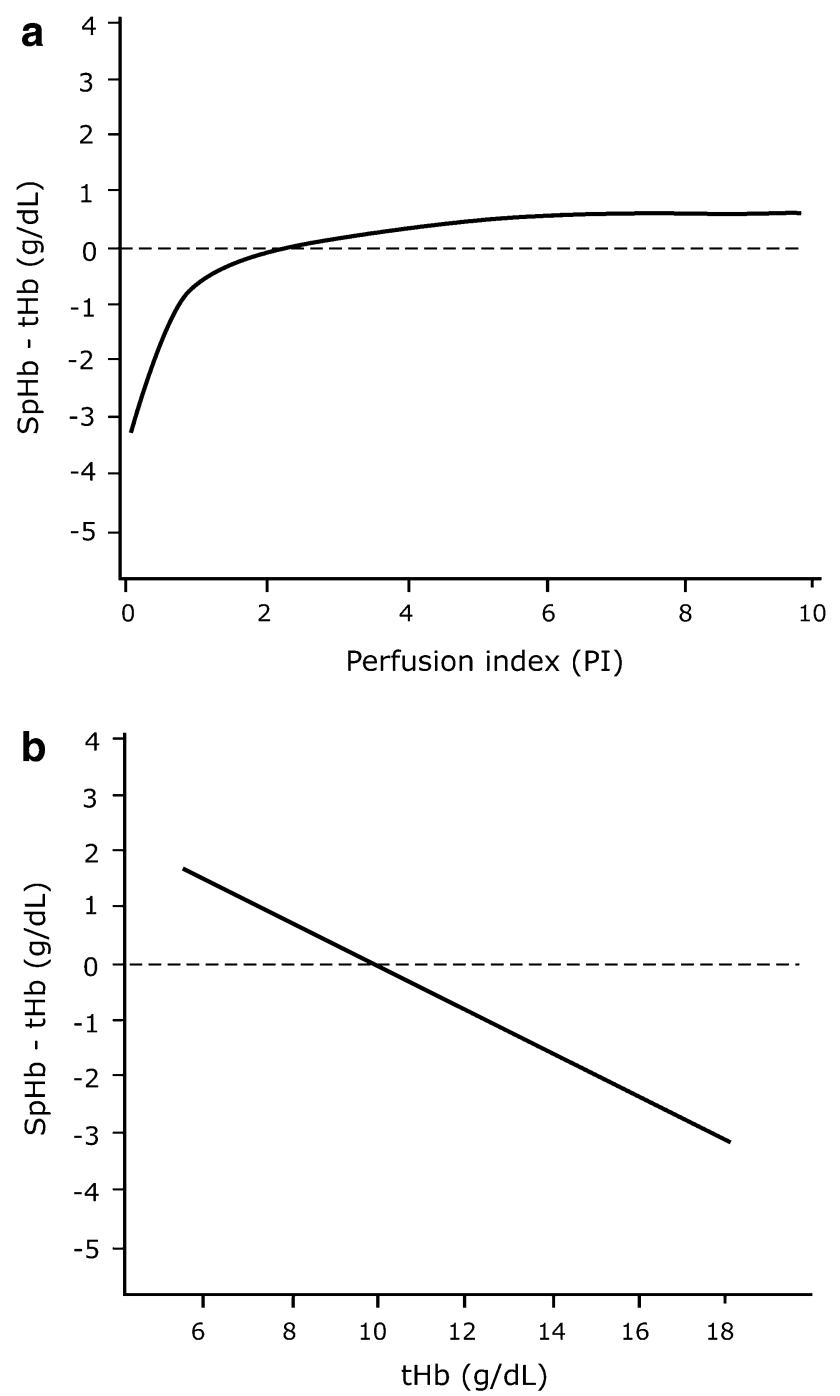

Fig. 1 The relationship between bias $(\mathrm{SpHb}-\mathrm{tHb})$ and perfusion index (PI) or tHb. a The bias appears to increase to between 0 and 1 with a higher PI. The values of bias and PI are from Refs. [24, 26, 29]. The trend is illustrated by a nonparametric smoothed regression line. b The bias appears to be inversely correlated with higher tHb. The values of bias and $\mathrm{tHb}$ are from Refs. [5, 20, 24]

\section{Hemoglobin Synthesis Disorders}

Hemoglobin synthesis disorders, such as thalassemia, Hb c, and sickle cell disease, may affect the $\mathrm{SpHb}$ value. One study of patients with sickle cell disease using the Pronto-7 demonstrated a moderate correlation with $\mathrm{SpHb}$ and $\mathrm{tHb}$ [44]. However, that study showed a wide range of bias $(-4.8$ to $4.5 \mathrm{~g} / \mathrm{dL})$, and $\mathrm{SpHb}$ values were better correlated when the $\mathrm{tHb}$ level was $9-11 \mathrm{~g} / \mathrm{dL}$. The authors concluded that this noninvasive method is appropriate for monitoring $\mathrm{Hb}$ changes rather than performing spot measurements in an individual.
Improper Application

Improper probe size compared with finger size, improper probe positioning, and motion can influence $\mathrm{SpHb}$ values. External fingernail coloring also affects the values. The Pronto-7 uses finger size to select an appropriate sensor size. When the Radical-7 is used, a light shield should be applied to avoid light interference. During general anesthesia, there is no concern regarding the motion of the probe. However, when patients are awake, such as in the emergency room, ICU, or those under spinal anesthesia, movement of the probe can affect the availability and accuracy of data.

\section{Others}

Other factors can affect the measurements; these include intravascular dyes (indocyanin or methylene blue), elevated bilirubin, low arterial saturation, and electrical interference.

\section{Clinical Applications and Further Study}

$\mathrm{SpHb}$ is useful for trend monitoring rather than for transfusion decision making, due to its limited accuracy. Predicting the change in $\mathrm{Hb}$ can facilitate preparation for adverse events or a transfusion. The concept of in vivo adjustment can increase the accuracy of performance of $\mathrm{SpHb}$ [41]. If the actual $\mathrm{tHb}$ is determined by drawing blood at baseline, then the difference between $\mathrm{SpHb}$ and $\mathrm{tHb}$ at baseline could be used to estimate $\mathrm{tHb}$ at other times. The accuracy of $\mathrm{SpHb}$ is insufficient to substitute for invasive $\mathrm{Hb}$ monitoring. However, it has undeniable advantages of noninvasiveness and continuous and immediate data availability. Most published studies considered the accuracy of devices. However, more data are required if $\mathrm{SpHb}$ is to be used to improve clinical outcomes.

\section{Conclusion}

Noninvasive continuous hemoglobin monitoring shows promise in terms of improving patient care and outcomes in various healthcare settings. However, many factors influence its accuracy, which must be further improved for use in patients with unstable hemodynamics or with bleeding. We suggest that devices for continuous hemoglobin monitoring can be used as a supplemental tool for monitoring of blood loss during anesthesia. However, SpHb cannot substitute for an invasive $\mathrm{Hb}$ measurement until its accuracy is as good as that of the blood-drawn reference value. 


\section{Compliance with Ethics Guidelines}

Conflict of Interest Ji-Hyun Lee, Yong-Hee Park, and Jin-Tae Kim declare that they have no conflict of interest.

Human and Animal Rights and Informed Consent This article does not contain any studies with human or animal subjects performed by any of the authors.

\section{References}

Papers of particular interest, published recently, have been highlighted as:

- Of importance

- Of major importance

1. Severinghaus JW, Koh SO. Effect of anemia on pulse oximeter accuracy at low saturation. J Clin Monit. 1990;6(2):85-8.

2. Barker SJ, Badal JJ. The measurement of dyshemoglobins and total hemoglobin by pulse oximetry. Curr Opin Anaesthesiol. 2008;21(6):805-10.

3. - Lindner G, Exadaktylos AK. How noninvasive haemoglobin measurement with pulse CO-oximetry can change your practice: an expert review. Emerg Med Int. 2013;2013:701529. The present review gives an overview on the technology itself and reviews the current literatures evaluated the accuracy of pulse $\mathrm{CO}$ oximetry in operating room, emergency room and outpatients.

4. Coquin J, Bertarrex A, Dewitte A, Lefevre L, Joannes-Boyau O, Fleureau C, Winnock S, Leuillet S, Janvier G, Ouattara A. Accuracy of determining hemoglobin level using occlusion spectroscopy in patients with severe gastrointestinal bleeding. Anesthesiology. 2013;118(3):640-8.

5. Gayat E, Aulagnier J, Matthieu E, Boisson M, Fischler M. Noninvasive measurement of hemoglobin: assessment of two different point-of-care technologies. PLoS One. 2012;7(1):e30065.

6. Amir O, Weinstein D, Zilberman S, Less M, Perl-Treves D, Primack H, Weinstein A, Gabis E, Fikhte B, Karasik A. Continuous noninvasive glucose monitoring technology based on "occlusion spectroscopy". J Diabetes Sci Technol. 2007;1(4):463-9.

7. Noiri E, Kobayashi N, Takamura Y, Iijima T, Takagi T, Doi K, Nakao A, Yamamoto T, Takeda S, Fujita T. Pulse total-hemoglobinometer provides accurate noninvasive monitoring. Crit Care Med. 2005;33(12):2831-5.

8. Rabe H, Alvarez RF, Whitfield T, Lawson F, Jungmann H. Spectroscopic noninvasive measurement of hemoglobin compared with capillary and venous values in neonates. Neonatology. 2010;98(1):1-5.

9. Rabe H, Stupp N, Ozgun M, Harms E, Jungmann H. Measurement of transcutaneous hemoglobin concentration by noninvasive white-light spectroscopy in infants. Pediatrics. 2005;116(4): $841-3$.

10. $\bullet$ Morey TE, Gravenstein N, Rice MJ. Assessing point-of-care hemoglobin measurement: be careful we don't bias with bias. Anesth Analg. 2011;113(6):1289-91. The previous evaluations of accuracy of pulse CO-oximetry have some limitation according to the analyzing method. Pitfalls of Bland-Altman method and hemoglobin range selection bias might be act as a new bias. The advantage of rapidity of point-of-care hemoglobin measurement should be balanced against the accuracy of data.

11. Perrino AC Jr, O'Connor T, Luther M. Transtracheal Doppler cardiac output monitoring: comparison to thermodilution during noncardiac surgery. Anesth Analg. 1994;78(6):1060-6.
12. Critchley LA, Lee A, Ho AM. A critical review of the ability of continuous cardiac output monitors to measure trends in cardiac output. Anesth Analg. 2010;111(5):1180-92.

13. • Colquhoun DA, Forkin KT, Durieux ME, Thiele RH. Ability of the Masimo pulse CO-oximeter to detect changes in hemoglobin. J Clin Monit Comput. 2012;26(2):69-73. The trending ability of pulse Co-oximeter was analyzed by the four quadrant plot technique, testing directionality, and Critchley's polar plot method in 24 patients undergoing major thoracic and lumbar spine surgery. The pulse CO-oximetry offers an acceptable trend monitor in this study.

14. Morey TE, Gravenstein N, Rice MJ. Let's think clinically instead of mathematically about device accuracy. Anesth Analg. 2011; 113(1):89-91.

15. - Rice MJ, Gravenstein N, Morey TE. Noninvasive hemoglobin monitoring: how accurate is enough? Anesth Analg. 2013;117(4): 902-7. The recent publications on the accuracy of pulse COoximeter focusing on the traditional statistical metrics of bias and precision are not enough to guide decision making for transfusion. The hemoglobin error grid with the data of $\mathrm{Hb}$ range of 6-10 g/dL should be used to evaluation of clinical usefulness of the noninvasive $\mathrm{Hb}$ monitoring.

16. Park YH, Lee JH, Song HG, Byon HJ, Kim HS, Kim JT. The accuracy of noninvasive hemoglobin monitoring using the radical-7 pulse CO-oximeter in children undergoing neurosurgery. Anesth Analg. 2012;115(6):1302-7.

17. Dewhirst E, Naguib A, Winch P, Rice J, Galantowicz M, McConnell P, Tobias JD. Accuracy of noninvasive and continuous hemoglobin measurement by pulse CO-oximetry during preoperative phlebotomy. J Intensive Care Med. 2013;29:238-42.

18. Jung YH, Lee J, Kim HS, Shin SH, Sohn JA, Kim EK, Choi JH. The efficacy of noninvasive hemoglobin measurement by pulse CO-oximetry in neonates. Pediatr Crit Care Med. 2013;14(1): $70-3$.

19. Agrawal A, Beethe AB, Sullivan JN, Jones BM, Adams JJ, Duhacheck-Stapleman AL. Continuous hemoglobin monitoring during massive blood transfusion in a multivisceral pediatric transplant patient. J Clin Anesth. 2013;25:578-81.

20. Causey MW, Miller S, Foster A, Beekley A, Zenger D, Martin M. Validation of noninvasive hemoglobin measurements using the Masimo Radical-7 SpHb Station. Am J Surg. 2011;201(5): $592-8$.

21. - Applegate RL 2nd, Barr SJ, Collier CE, Rook JL, Mangus DB, Allard MW. Evaluation of pulse cooximetry in patients undergoing abdominal or pelvic surgery. Anesthesiology. 2012;116(1): 65-72. The bias was analyzed based on subgroups defined for patients' and intraoperative characteristics. The amount of blood loss, hemoglobin level, intraoperative transfusion, and the level of anesthesia influenced on bias.

22. Lamhaut L, Apriotesei R, Combes X, Lejay M, Carli P, Vivien B. Comparison of the accuracy of noninvasive hemoglobin monitoring by spectrophotometry $(\mathrm{SpHb})$ and $\mathrm{HemoCue}(\mathrm{R})$ with automated laboratory hemoglobin measurement. Anesthesiology. 2011;115(3):548-54.

23. Butwick A, Hilton G, Carvalho B. Non-invasive haemoglobin measurement in patients undergoing elective Caesarean section. Br J Anaesth. 2012;108(2):271-7.

24. Berkow L, Rotolo S, Mirski E. Continuous noninvasive hemoglobin monitoring during complex spine surgery. Anesth Analg. 2011;113(6):1396-402.

25. Miller RD, Ward TA, Shiboski SC, Cohen NH. A comparison of three methods of hemoglobin monitoring in patients undergoing spine surgery. Anesth Analg. 2011;112(4):858-63.

26. Gayat E, Bodin A, Sportiello C, Boisson M, Dreyfus JF, Mathieu E, Fischler M. Performance evaluation of a noninvasive hemoglobin monitoring device. Ann Emerg Med. 2011;57(4):330-3. 
27. Sjostrand F, Rodhe P, Berglund E, Lundstrom N, Svensen C. The use of a noninvasive hemoglobin monitor for volume kinetic analysis in an emergency room setting. Anesth Analg. 2013;116(2):337-42.

28. Moore LJ, Wade CE, Vincent L, Podbielski J, Camp E, Junco DD, Radhakrishnan H, McCarthy J, Gill B, Holcomb JB. Evaluation of noninvasive hemoglobin measurements in trauma patients. Am J Surg. 2013;206(6):1041-7.

29. Frasca D, Dahyot-Fizelier C, Catherine K, Levrat Q, Debaene B, Mimoz O. Accuracy of a continuous noninvasive hemoglobin monitor in intensive care unit patients. Crit Care Med. 2011;39(10):2277-82.

30. Joseph B, Hadjizacharia P, Aziz H, Snyder K, Wynne J, Kulvatunyou N, Tang A, O'Keeffe T, Latifi R, Friese R, et al. Continuous noninvasive hemoglobin monitor from pulse ox: ready for prime time? World J Surg. 2013;37(3):525-9.

31. Nguyen BV, Vincent JL, Nowak E, Coat M, Paleiron N, Gouny P, Ould-Ahmed M, Guillouet M, Arvieux CC, Gueret G. The accuracy of noninvasive hemoglobin measurement by multiwavelength pulse oximetry after cardiac surgery. Anesth Analg. 2011;113(5):1052-7.

32. - Coquin J, Dewitte A, Manach YL, Caujolle M, Joannes-Boyau O, Fleureau C, Janvier G, Ouattara A. Precision of noninvasive hemoglobin-level measurement by pulse $\mathrm{CO}$-oximetry in patients admitted to intensive care units for severe gastrointestinal bleeds. Crit Care Med. 2012;40(9):2576-82. The accuracy of pulse COoximetry was evaluated in 33 patients with gastrointestinal bleeding in intensive care unit. The proportion of inaccurate measurements was higher for pulse CO-oximeter measurements and the use of norepinephrine also increased the unavailability of measurements (42 vs. 15\%). This study conclude that transfusion decision based on $\mathrm{SpHb}$ is potentially hazardous.

33. Macknet MR, Allard M, Applegate RL II, Rook J. The accuracy of noninvasive and continuous total hemoglobin measurement by pulse CO-oximetry in human subjects undergoing hemodilution. Anesth Analg. 2010;111(6):1424-6.

34. Shah N, Osea EA, Martinez GJ. Accuracy of noninvasive hemoglobin and invasive point-of-care hemoglobin testing compared with a laboratory analyzer. Int J Lab Hematol. 2013. doi:10.1111/ijlh.12118.
35. Kim MJ, Park Q, Kim MH, Shin JW, Kim HO. Comparison of the accuracy of noninvasive hemoglobin sensor (NBM-200) and portable hemoglobinometer (HemoCue) with an automated hematology analyzer (LH500) in blood donor screening. Ann Lab Med. 2013;33(4):261-7.

36. Belardinelli A, Benni M, Tazzari PL, Pagliaro P. Noninvasive methods for haemoglobin screening in prospective blood donors. Vox Sang. 2013;105(2):116-20.

37. Pinto M, Barjas-Castro ML, Nascimento S, Falconi MA, Zulli R, Castro V. The new noninvasive occlusion spectroscopy hemoglobin measurement method: a reliable and easy anemia screening test for blood donors. Transfusion. 2013;53(4):766-9.

38. Hadar E, Raban O, Bouganim T, Tenenbaum-Gavish K, Hod M. Precision and accuracy of noninvasive hemoglobin measurements during pregnancy. J Matern Fetal Neonatal Med. 2012;25(12): 2503-6.

39. Yamada H, Saeki M, Ito J, Kawada K, Higurashi A, Funakoshi H, Takeda K. The relative trending accuracy of noninvasive continuous hemoglobin monitoring during hemodialysis in critically ill patients. J Clin Monit Comput. 2014. doi:10.1007/s10877-0149574-6.

40. Miller RD, Ward TA, McCulloch CE, Cohen NH. Does a digital regional nerve block improve the accuracy of noninvasive hemoglobin monitoring? J Anesth. 2012;26(6):845-50.

41. Isosu T, Obara S, Hosono A, Ohashi S, Nakano Y, Imaizumi T, Mogami M, Murakawa M. Validation of continuous and noninvasive hemoglobin monitoring by pulse CO-oximetry in Japanese surgical patients. J Clin Monit Comput. 2013;27(1):55-60.

42. Bergek C, Zdolsek JH, Hahn RG. Accuracy of noninvasive haemoglobin measurement by pulse oximetry depends on the type of infusion fluid. Eur J Anaesthesiol. 2013;30(2):73-9.

43. Hahn RG, Li Y, Zdolsek J. Non-invasive monitoring of blood haemoglobin for analysis of fluid volume kinetics. Acta Anaesthesiol Scand. 2010;54(10):1233-40.

44. Al-Khabori M, Al-Hashim A, Jabeen Z, Al-Farsi K, Al-Huneini M, Al-Riyami A, Al-Kemyani N, Daar S. Validation of a noninvasive pulse CO-oximetry-based hemoglobin estimation in patients with sickle cell disease. Int J Lab Hematol. 2013; 35(5):e21-3. 